\title{
Ciliogenesis is Not Directly Regulated by LRRK2 Kinase Activity in Neurons
}

\author{
Hyejung Kim ${ }^{1 \dagger}$, Hyuna Sim ${ }^{2,3 \dagger}$, Joo-Eun Lee ${ }^{2}$, Mi Kyoung Seo ${ }^{4}$, Juhee Lim ${ }^{5 \ddagger}$, Yeojin Bang ${ }^{5}$, \\ Daleum Nam ${ }^{1}$, Seo-Young Lee ${ }^{6}$, Sun-Ku Chung ${ }^{7}$, Hyun Jin Choi ${ }^{5}$, Sung Woo Park ${ }^{4,8}$, \\ Ilhong Son ${ }^{1,9}$, Janghwan Kim ${ }^{2,3 *}$ and Wongi Seol ${ }^{1 *}$ \\ ${ }^{1}$ InAm Neuroscience Research Center, Sanbon Medical Center, College of Medicine, Wonkwang University, Gunpo 15865, \\ ${ }^{2}$ Stem Cell Convergence Research Center, Korea Research Institute of Bioscience and Biotechnology (KRIBB), Daejeon 34141, \\ ${ }^{3}$ Department of Functional Genomics, KRIBB School of Bioscience, University of Science and Technology, Daejeon 34141, \\ ${ }^{4}$ Paik Institute for Clinical Research, Inje University College of Medicine, Busan 47392, ${ }^{5}$ College of Pharmacy, CHA University, \\ Seongnam 13496, ${ }^{6}$ Division of Clinical Medicine, Korea Institute of Oriental Medicine, Daejeon 34054, ${ }^{7}$ Division of Herbal \\ Medicine Research, Korea Institute of Oriental Medicine, Daejeon 34054, ${ }^{8}$ Department of Convergence Biomedical Science, \\ Inje University College of Medicine, Busan 47392, ${ }^{9}$ Department of Neurology, Sanbon Medical Center, College of Medicine, \\ Wonkwang University, Gunpo 15865, Korea

\begin{abstract}
Mutations in the Leucine-rich repeat kinase 2 (LRRK2) gene are the most prevalent cause of familial Parkinson's disease (PD). The increase in LRRK2 kinase activity observed in the pathogenic G2019S mutation is important for PD development. Several studies have reported that increased LRRK2 kinase activity and treatment with LRRK2 kinase inhibitors decreased and increased ciliogenesis, respectively, in mouse embryonic fibroblasts (MEFs) and retinal pigment epithelium (RPE) cells. In contrast, treatment of SH-SY5Y dopaminergic neuronal cells with PD-causing chemicals increased ciliogenesis. Because these reports were somewhat contradictory, we tested the effect of LRRK2 kinase activity on ciliogenesis in neurons. In SH-SY5Y cells, LRRK2 inhibitor treatment slightly increased ciliogenesis, but serum starvation showed no increase. In rat primary neurons, LRRK2 inhibitor treatment repeatedly showed no significant change. Little difference was observed between primary cortical neurons

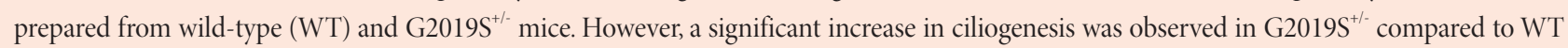
human fibroblasts, and this pattern was maintained in neural stem cells (NSCs) differentiated from the induced pluripotent stem cells (iPSCs) prepared from the same WT/G2019S fibroblast pair. NSCs differentiated from G2019S and its gene-corrected WT counterpart iPSCs were also used to test ciliogenesis in an isogenic background. The results showed no significant difference between WT and G2019S regardless of kinase inhibitor treatment and B27-deprivation-mimicking serum starvation. These results suggest that LRRK2 kinase activity may be not a direct regulator of ciliogenesis and ciliogenesis varies depending upon the cell type or genetic background.
\end{abstract}

Key words: Primary cilia, Neuron, LRRK2 kinase, Ciliogenesis, Parkinson’s disease

\section{INTRODUCTION}

Submitted March 4, 2021, Revised April 28, 2021,

Accepted May 14, 2021

* To whom correspondence should be addressed. Janghwan Kim, TEL: 82-42-860-4478, FAX: 82-42-860-4608 e-mail: janghwan.kim@kribb.re.kr

Wongi Seol, TEL: 82-31-390-2411, FAX: 82-31-390-2414

e-mail:wseolha@gmail.com

${ }^{\top}$ These authors contributed equally to this work.

Present address: College of Pharmacy, Woosuk University, Wanju 55338, Korea.
Several mutations in the leucine-rich repeat kinase 2 (LRRK2) gene cause Parkinson's disease (PD), the second most common neurodegenerative disease $[1,2]$. Among the $L R R K 2$ pathogenic mutations, the G2019S pathogenic mutation is the most prevalent [3] and increases kinase activity [4]. The G2019S mutation was frequently found in sporadic as well as familial PD cases [5]. The properties of the G2019S mutation have led to intensive studies on the kinase activity of LRRK2 in hopes of developing PD therapeu-
Copyright ( $)$ Experimental Neurobiology 2021. www.enjournal.org
This is an Open Access article distributed under the terms of the Creative Commons Attribution Non-Commercial License (http://creativecommons.org/licenses/by-nc/4.0) which permits unrestricted non-commercial use, distribution, and reproduction in any medium, provided the original work is properly cited. 
tics [6].

Cilia are cellular appendages that protrude from the cell body and are found in most cells including neurons. Primary cilia in neurons play roles in modulating neurogenesis such as axonal guidance and cell polarity [7]. Functional cilia are known to be critical for the development of midbrain dopaminergic neurons via Sonic hedgehog (Shh)-mediated signaling [8]. Serum starvation is one of the major cues inducing ciliogenesis, the formation of primary cilia [9].

LRRK2 specifically phosphorylates a subset of Rab proteins, major regulators of vesicle trafficking $[10,11]$, which is essential for ciliogenesis. In addition, Rab8 and Rab10 are crucial regulators of ciliogenesis $[12,13]$. Recent studies reported that the kinase activity increased by LRRK2 pathogenic R1441C/G and G2019S mutations impaired ciliogenesis via Rab8/10 phosphorylation [11, 14]. However, an earlier study showed that only Rab10 knockout (KO), but not Rab8 KO, increased ciliogenesis although both Rab8 and 10 are substrates of LRRK2 kinase [14]. Further studies disclosed that PPM1H specifically dephosphorylated Rab proteins phosphorylated by LRRK2, and PPM1H knockdown suppressed ciliogenesis [15]. Sobu et al. [16] reported that treatment of pathogenic LRRK2 R1441C MEF cells with MLi-2, an LRRK2 kinase inhibitor, significantly increased ciliogenesis at least 3 -fold. Another study reported that the proportion of ciliated cells in HEK293T cells expressing LRRK2 pathogenic mutants Y1699C, G2019S, and R1441C, but not WT, was decreased and that MLi-2 treatment partially rescued ciliogenesis defects in the same cells expressing mutants only under serum-fed conditions [17]. A recent study reported that LRRK2-indcued Rab10 phosphorylation regulated the myosin Va-RILP2 complex to inhibit cilia formation [18].

The SH-SY5Y cell line is a widely used cellular model of dopaminergic neurons [19]. Bae et al. [20] reported that treatment of SHSY5Y and RPE cells with mitochondrial respiratory complex-1 inhibitors, rotenone and 1-methyl-4-phenylpyridinium $\left(\mathrm{MPP}^{+}\right)$, which are also PD-causing chemicals, increased ciliogenesis and cilium length. They further showed that a decrease in mitochondrial fission by siDrp1 or fusion by siOPA1, decreased or increased ciliogenesis and cilium length, respectively [20]. They concluded that primary cilia modulate mitochondrial stress to enhance dopaminergic neuron survival. Because both an increase in LRRK2 kinase activity and cellular exposure to $\mathrm{MPP}^{+}$and rotenone are major causes of PD development, the results of these studies are somewhat contradictory $[11,14,15,17,20]$. Except for the study by Bae et. al, most of these studies were carried out in non-neuronal cells such as MEF, hTERT-RPE, iPSCs, A549, and HEK-293T cells, although the immunostainings of cilia in in brain tissue neurons [11] and primary astrocytes [17] have been reported.
Therefore, we decided to test the effect of LRRK2 kinase activity on ciliogenesis in cell models such as primary neurons and neural stem cells (NSCs) differentiated from human induced pluripotent stem cells (iPSCs) derived from fibroblasts obtained from a PD patient with the G2019S mutation as well as differentiated SH-SY5Y cells. Our results suggest that the relationship between LRRK2 kinase activity and ciliogenesis is dependent upon the cell type or genetic background, and is much more complicated than presented in previous reports.

\section{MATERIALS AND METHODS}

\section{Fibroblast culture}

Human fibroblasts from a PD patient harboring the LRRK2 G2019S mutation (ND38262) and from a healthy control subject (MRC5) were purchased from the Coriell Institute for Medical Research (Camden, NJ, USA) and the American Type Culture Collection (ATCC, Manassas, VA, USA), respectively. They were cultured in Minimal Essential Medium (MEM) with 10\% fetal bovine serum (FBS), 1\% MEM non-Essential amino acids Solution (100X), $1 \%$ sodium pyruvate $(100 \mathrm{mM})$, and $1 \%$ penicillin-streptomycin (P/S, Thermo Fisher Scientific, Waltham, MA, USA). Our research samples were maintained according to the manufacturer's recommendations and the study was determined to be exempt from Institutional Review Board (IRB) review.

\section{Differentiation of human induced pluripotent stem cells into neural stem cells}

Human iPSCs were generated from WT (MRC5, ATCC ${ }^{\circledR}$ CCL171) and $\mathrm{G}^{2019 S^{+/}}$(ND38262, Coriell) fibroblasts using episomal vectors and electroporation with the Neon transfection system (Invitrogen, Carlsbad, CA, USA) as described previously [21]. Established human iPSCs were fed TeSR ${ }^{\mathrm{TM}}$-E8 ${ }^{\mathrm{TM}}$ (STEMCELL Technologies, Vancouver, BC, Canada) according to the manufacturer's recommendations. Neural stem cells (NSCs) were differentiated from human iPSCs as previously described [22] with some modifications [23]. Human iPSCs were seeded on GeltrexTM LDEVfree Reduced Growth Factor Basement Membrane Matrix-coated dishes at about $20 \%$ confluency. For neural induction, Neural Induction Basal Medium 1 [NIM: 50\% Advanced DMEM/F12, $50 \%$ Neurobasal medium, N-2 supplement (100X), B-27 supplement (50X) minus vitamin A (B27), $1 \%$ Glutamax $^{\mathrm{TM}}$, and 1\% P/S] supplemented with $10 \mathrm{ng} / \mathrm{ml}$ human LIF (PeproTech, Inc., Rocky

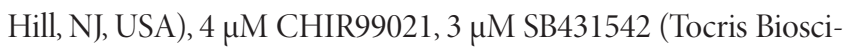
ence, Bristol, UK), $2 \mu \mathrm{M}$ Dorsomorphin, and $0.1 \mu \mathrm{M}$ Compound E (Sigma-Aldrich, St. Louis, MO, USA) were used to treat the cells for two days. Then, the differentiating cells were fed with the 
same medium without Dorsomorphin for another five days. On day 7 of differentiation, the cells were passaged with Accutase cell detachment solution (Sigma-Aldrich) and maintained in NIM supplemented with $10 \mathrm{ng} / \mathrm{ml}$ human LIF, 3 MM CHIR99021, and $2 \mu \mathrm{M}$ SB431542. NSCs were passaged every week using Accutase cell detachment solution. The NSCs were treated with $1 \mu \mathrm{M}$ MLi2 (MedChemExpress, NJ, USA) or dimethyl sulfoxide (DMSO, Sigma-Aldrich) for control cells for three days before the detection of ciliogenesis.

The NSCs were further differentiated into neuronal cells as previously described [24] with some modifications. The NSCs were plated on poly-L-ornithine (PLO)/laminin (Sigma-Aldrich)coated dishes. Two days after seeding, the medium was changed to neuronal differentiation medium (NDM: Neurobasal medium, B-27, 1\% Glutamax ${ }^{\mathrm{TM}}$ supplement, and 1\% P/S) supplemented with $20 \mathrm{ng} / \mathrm{ml}$ brain-derived neurotrophic factor (BDNF, Research and Diagnostic Systems, Inc., Minneapolis, MN, USA), 20 ng/ $\mathrm{ml}$ glial cell-derived neurotrophic factor (GDNF, Research and Diagnostic Systems, Inc.), 0.2 mM 2-phospho ascorbic acid, 400 $\mu \mathrm{M}$ dibutyryl-cAMP (Enzo Life Sciences, NY, USA) and $1 \mathrm{ng} / \mathrm{mL}$ transforming growth factor $\beta$-3 (TGF- $\beta 3$, PeproTech, Inc., Rocky Hill, NJ, USA). Half of the medium volume was changed every three days. On day 14 of the differentiation, the cells were re-plated on PLO/laminin-coated dishes. On day 18 of the differentiation, the cells were treated with $1 \mu \mathrm{M}$ MLi- 2 or DMSO control for $24 \mathrm{~h}$ before the detection of ciliogenesis.

The PD patient's B-lymphocytes with an LRRK2 G2019S mutation (ND14317, Coriell) were reprogrammed into G2019S iPSCs [21] and then, the G2019S mutation was corrected to generate the KIOMi002-A WT iPSC line to prepare an isogenic WT/G2019S pair [25]. The WT/G2019S pair of iPSCs were incubated and treated with $400 \mathrm{nM}$ MLi-2 for 24 h. The same WT/G2019S pair of iPSCs were differentiated to NSCs as above, and cultured with or without B27 for $24 \mathrm{~h}$. Both cell types were stained with antiArl13b (Proteintech, \# 17711-1-AP) to detect cilia as described below.

\section{Culture of primary neurons, SH-SY5Y, and SN4741 cells}

Pregnant Sprague-Dawley rats were obtained from Orient Bio (Seongnam, South Korea) and the experimental procedures were approved by the Inje Medical College Committee for Animal Experimentation and the Institutional Animal Laboratory Review Board (Approval no. 2018-016).

Cortical or hippocampal neuronal cultures were prepared from the cortex or hippocampus, respectively, of fetuses on embryonic day 19 as previously described [26]. To test for ciliogenesis, the cells were plated on 12 -well dishes at a density of $1 \times 10^{5}$ cells per well and the culture medium with $1 \mathrm{X}$ anti-mitotic agent $(80 \mu \mathrm{M}$ 5'fluoro-2' deoxyuridine and $40 \mu \mathrm{M}$ uridine) was changed every two days. After incubation for 14 days, the cells were treated with $1 \mu \mathrm{M}$ GSK-2578215A (GSK) or $100 \mathrm{nM}$ MLi-2 for one day before immunostaining for ciliogenesis analysis.

$\mathrm{G}_{2019 S^{+/-}}$mice were generated from crossings G2019S [FVB/ N-Tg(LRRK2*G2019S)1Cjli/J; The Jackson Lab. No: 009609] and WT mice [FVB/NJ; The Jackson Lab. No: 001800]. We were able to obtain only two $\mathrm{G}^{2} 219 \mathrm{~S}^{+/-}$mice by two independent crossings. After genotyping, murine cortical neurons were prepared from $\mathrm{G} 019 \mathrm{~S}^{+/-}$and their WT littermate mice using a method similar to that used for rat primary neuronal culture (IACUC approval no. 190095) and grown in Neurobasal medium with 1\% L-glutamine, $2 \% \mathrm{~B} 27$, and $1 \% \mathrm{P} / \mathrm{S}$ for nine days. Then, the cells were incubated with or without $\mathrm{B} 27$, for $24 \mathrm{~h}$ and subjected to immunostaining.

SH-SY5Y cells were cultured in DMEM (Corning, \#10-013-CV) with $10 \% \mathrm{FBS}$ and $1 \% \mathrm{P} / \mathrm{S}$, and differentiated with $13 \mu \mathrm{M}$ retinoic acid for six days. The differentiated SH-SH5Y (dSH-SY5Y) cells were treated with MLi-2 for $24 \mathrm{~h}$ before immunostaining. SN4741 dopaminergic neuronal cells were cultured in DMEM with 10\% FBS and $1 \% \mathrm{P} / \mathrm{S}$ at $34^{\circ} \mathrm{C}$.

\section{Plasmids and transfection}

The pEGFP vector was used to construct pGFP-Rab10 expressing human Rab10 protein fused to GFP. SN4741 cells were transfected with pEGFP or GFP-Rab10 using Lipo-D293 (SignaGen Lab. Frederick, MD, USA). One day after transfection, the cells were serum-starved for $24 \mathrm{~h}$, fixed, and immunostained with the Arl13B antibody.

\section{Immunocytochemistry}

The cells were washed with Dulbecco's Phosphate-Buffered Saline (DPBS, Welgene, Korea) and fixed in 4\% formaldehyde (Electron Microscopy Sciences, Hatfield, PA, USA) for $10 \mathrm{~min}$ at room temperature. After washing with DPBS, the cells were blocked and permeabilized with 3\% BSA and 0.3\% TritonX-100 (SigmaAldrich) in DPBS for $1 \mathrm{~h}$ at room temperature.

The cells were stained with anti-Arl13b (1:100 250) in 1\% BSA in DPBS and washed with $0.1 \%$ BSA in DPBS, followed by incubation in the proper secondary antibody. If needed, anti- $\beta$ III tubulin (Merck Millipore, AB9354) was used for staining. Hoechst33342 was used to visualize the nuclei. Cell images were taken with a confocal microscope or a FLoid cell imaging station (Thermo Fisher Scientific) and the ciliated cells were counted by two persons blinded to the treatments. We used Arl13B as a cilia marker for the entire study. To obtain the percentage of ciliated cells, the number of Arl $13 \mathrm{~B}^{+}$cells was counted as ciliated cells and divided by the 
total number of cells.

\section{Western blot analysis}

To compare LRRK2 kinase activity, pT73-Rab10 antibody (Abcam, \#ab230261) was used after gel electrophoresis of cell lysates, as described previously [27]. In addition, $\beta$-actin (Santa-Cruz, \#sc47778) antibodies were used to normalize the loading amounts.

\section{Statistical analysis}

The Prism5 program (GraphPad Software, La joplla, CA, USA) was used to perform the statistical analyses. The data were analyzed by one-way ANOVA with Tukey's or Sidak's multiple comparison test. For experiments with two kinds of samples, the Student's $t$-test was applied. Significance was evaluated by $\mathrm{p}$-values and presented in graphs with the following indications: ${ }^{*} \mathrm{p}<0.05$, ${ }^{\star *} \mathrm{p}<0.01,{ }^{* *} \mathrm{p}<0.001,{ }^{* * *} \mathrm{p}<0.0001$. The data are expressed as the mean \pm SEM.

\section{RESULTS}

\section{Ciliogenesis in neurons is independent of LRRK2 kinase activity}

To investigate the effect of LRRK2 kinase activity on ciliogenesis in neurons, we first tested ciliogenesis in the dSH-SY5Y human dopaminergic neuronal cell line with or without serum starvation and treatment with MLi-2 (Fig. 1A). The dSH-SY5Y cells exhibited 50 60\% ciliogenesis. However, neither serum starvation nor MLi-2 treatment produced a distinct difference. In detail, serum starvation showed no increase in ciliogenesis, contrasting to previous study results using undifferentiated SH-SY5Y cells [20]. MLi2 treatment showed an increase as reported [14], but the increase was very weak at $\sim 1$-1-fold (Fig. 1A), compared to the $2 \sim 3$-fold increase previously reported in LRRK2 R1441C/G MEF cells [14, 16].

Because the result was different from the previous reports showing that MLi-2 treatment increased ciliogenesis in various cell
A

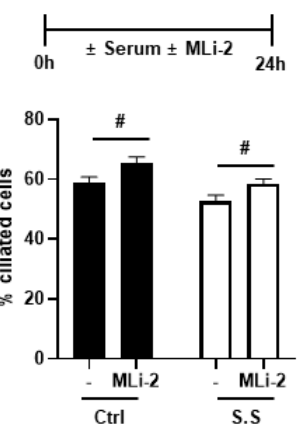

C
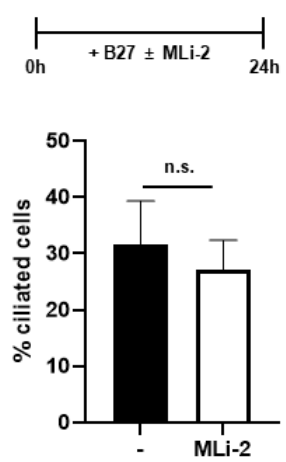
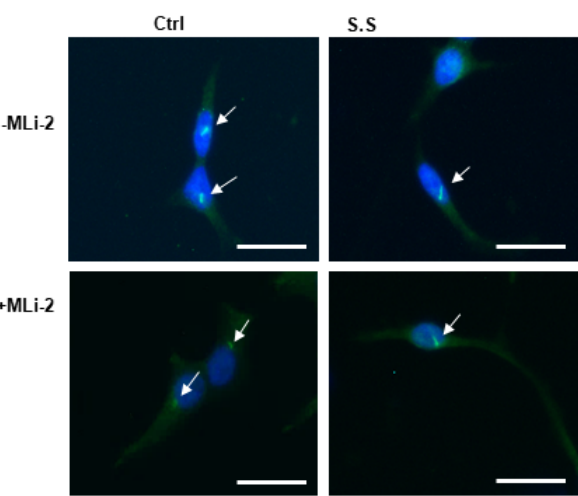

dSH-SY5Y

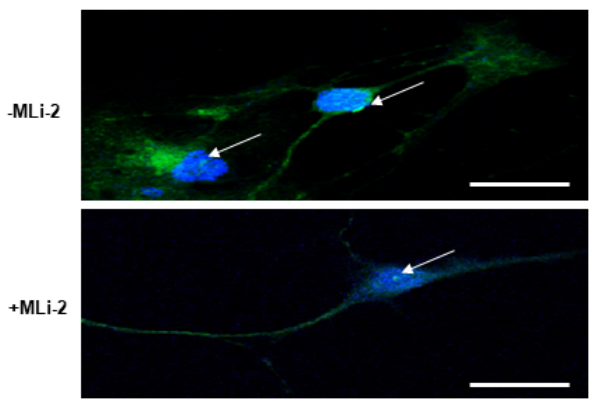

Rat hippocampal neurons
B
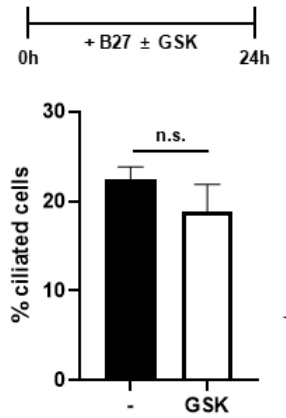

-GSK
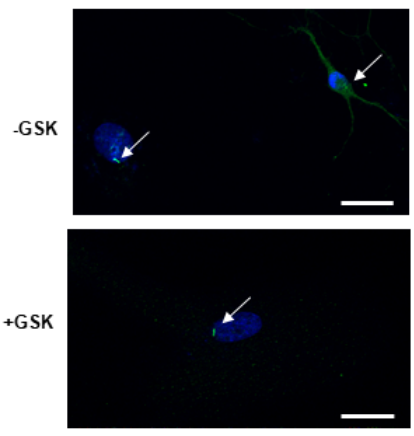

Rat cortical neurons
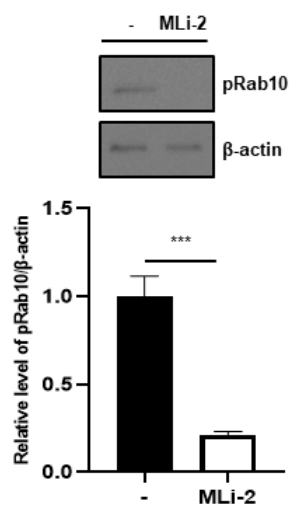

Fig. 1. Ciliogenesis in dSH-SY5Y cells and rat cortical neurons after MLi-2 treatment. Ciliated cells and nuclei were stained with Arl13B antibody and Hoechst 33342, respectively. Arl13B (green) cells were counted as ciliated cells and divided by the total number of cells to obtain the percentage of ciliated cells. In every cell image, the nuclei were stained with Hoechst 33342 (blue). (A) dSH-SY5Y cells incubated with DMSO (-) or $100 \mathrm{nM}$ MLi-2 under serum starvation (S.S) or normal (Ctrl) conditions for $24 \mathrm{~h}$. Scale bar: $20 \mu \mathrm{m}$. A total of 393 687 cells in nine images were analyzed. A summary graph and each representative image are shown. ${ }^{*} \mathrm{p}<0.05$ by the $t$-test. Primary rat cortical (B) and hippocampal (C) neurons treated with DMSO (-), 1 $\mu \mathrm{M}$ GSK-2578215A (GSK, B), or $100 \mathrm{nM} \mathrm{MLi-2} \mathrm{(C)} \mathrm{for} 24 \mathrm{~h}$. The treatment scheme, summary graph, and a representative image for each cell type are shown. Scale bar: $100 \mu \mathrm{m}$. A total of 81 92 cells in eleven images were analyzed. The white arrow indicates a ciliated cell. (D) The decrease in LRRK2 kinase activity in hippocampal neurons treated with MLi-2 was confirmed by a decrease in pRab10 levels. n.s.: not significant. 
types $[11,14]$, we tested rat cortical or hippocampal neurons with LRRK2 kinase inhibitors, GSK or MLi-2, respectively, under normal neuronal culture conditions. The primary neurons showed $22 \sim 31 \%$ ciliogenesis, but neither inhibitor treatment showed a significant difference compared to the untreated controls (Figs. 1B, 1C). The activity of MLi-2 as an LRRK2 kinase inhibitor was confirmed based on decreased levels of pT73-Rab10 in the corresponding cell lysates (Fig. 1D). We then tested rat cortical neurons with MLi-2 in the same culture conditions, but again, there was no significant difference (data not shown).

Because we could not observe the effect of the LRRK2 kinase inhibitor on ciliogenesis in neuronal cultures, we used primary cortical neurons prepared from heterozygous $\mathrm{G}_{2019 S^{+/}}$and their littermate WT mice, and directly measured the effect of LRRK2 kinase activity on ciliogenesis without kinase inhibitor. Primary neurons were prepared, cultured, and immunostained for both Arl13B and $\beta$ III tubulin, and the Arl13B ${ }^{+}$and $\beta$ III tubulin ${ }^{+}$cells were counted as neurons with cilia. There were $\sim 70 \sim 80 \%$ ciliated cells, similar to the previous reports [28,29], but there was little dif-

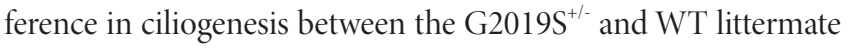
cells (Fig. 2A). Both serum starvation and autophagy have been reported as major inducers of ciliogenesis [9, 20, 30]. Although primary neuronal culture medium does not contain serum, the medium includes B27, a serum substitute $[31,32]$, for primary neuronal culture. To mimic serum-starvation excluding nutrients, we omitted the B27 supplement during the culture of these cortical neurons [33]. Our B27 deprivation was toxic to the neurons

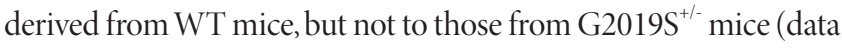
bot shown). The B27 deprivation exhibited no significant effect on the ciliogenesis of $\mathrm{G}_{2019 \mathrm{~S}^{+/}}$primary neurons (Fig. 2A). We

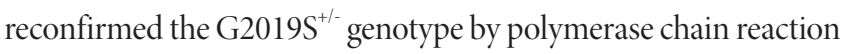
(PCR) analysis of tail DNA isolated from each mouse (Fig. 2B).

Taken together, the results we observed in primary neurons and dSH-SY5Y dopaminergic neuronal cells were different from the previous reports showing that LRRK2 kinase activity decreased ciliogenesis $[11,14,15,17]$.

\section{Ciliogenesis ratio of G2019S/WT varies depending upon the specific cell pairs compared}

To further test the effect of LRRK2 kinase activity on ciliogenesis, we used human fibroblasts originating from a PD patient with the G2019S mutation (ND38262) and WT MRC5 cells, a pair that has been used to produce neuroectodermal spheres by reprogramming technology [21]. Under serum-fed conditions, the G2019S
A

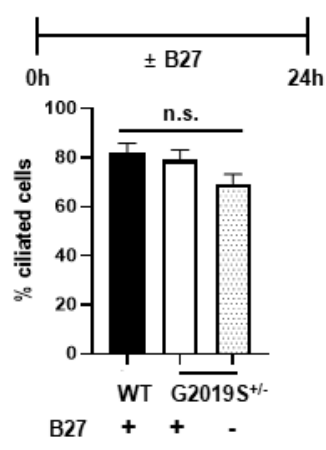

B

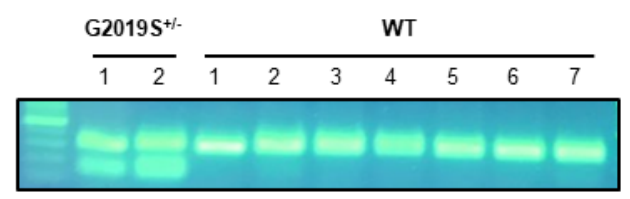

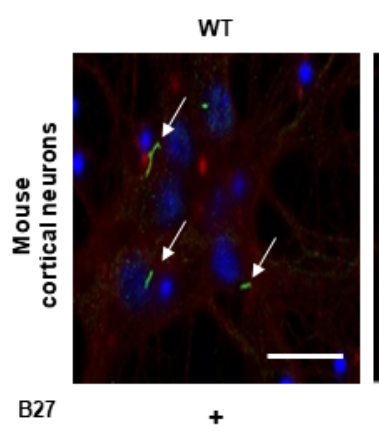

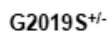
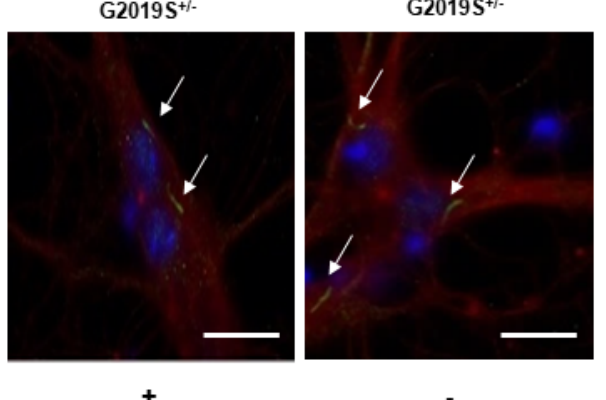

Fig. 2. Ciliogenesis of primary neurons derived from LRRK2 G2019S mice. Murine primary cortical neurons were prepared from LRRK2 G2019S ${ }^{+/}$ mice and WT littermates. Neurons were exposed to B27 deprivation to mimic serum starvation for 24 h on Day 9 . Ciliated neurons were confirmed by positive immunostaining with Arl13B (green) and $\beta \mathrm{III}$ tubulin (red). (A) The treatment scheme, summary graph, and a representative image for each cell type are shown. A total of 64 100 cells in 8 11 images were analyzed. Scale bar: $20 \mu \mathrm{m}$. The white arrow indicates a ciliated cell. (B) Genotyping of each littermate by PCR of tail DNA. 

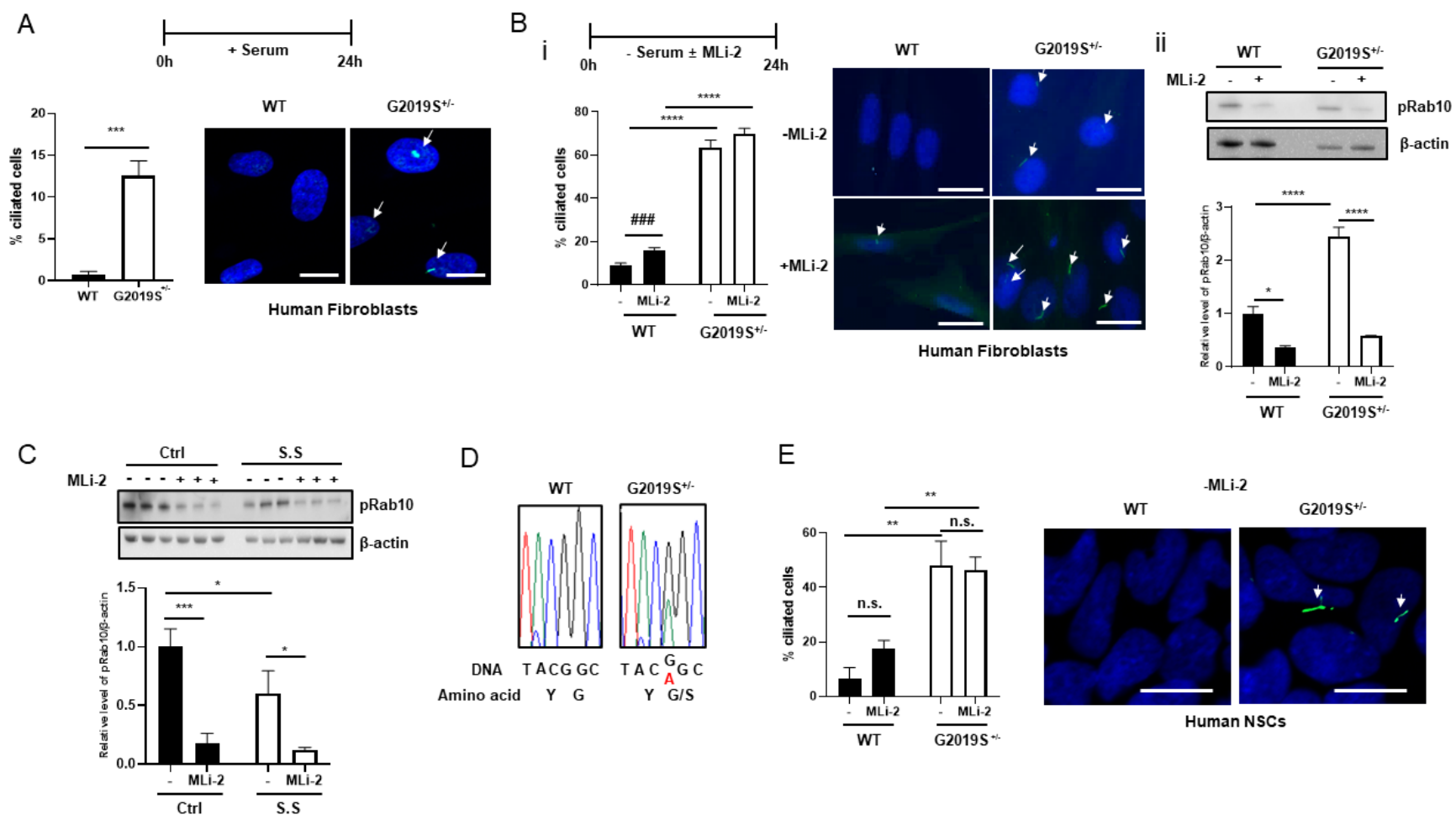

E
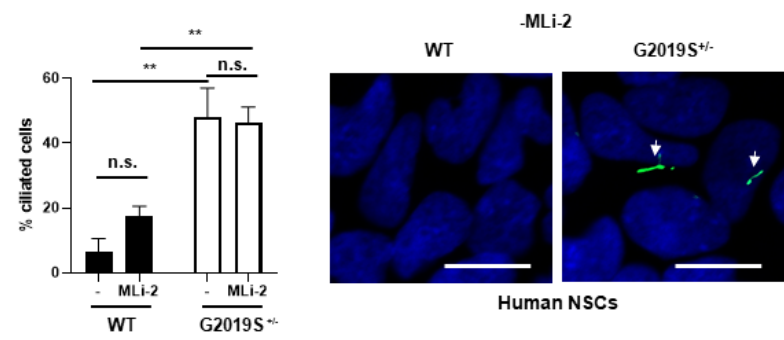

Fig. 3. Ciliogenesis of LRRK2 G2019S ${ }^{+/}$(ND38262) and WT (MRC5) fibroblasts (A D) and NSCs (E). All human fibroblast cells were cultured under serum-fed (A) or starved (B, C) conditions, and the Arl13B (green) ciliated cells were counted. (A) A total of 89 102 cells in five images were analyzed. (B) Ciliogenesis of fibroblasts after treatment with DMSO (control, -) or $100 \mathrm{nM} \mathrm{MLi-2} \mathrm{for} 24 \mathrm{~h}$. i) Summary graph and each representative image. A total of 370 518 cells in 6 7 images were analyzed. ii) The LRRK2 kinase activity of each sample was analyzed by Western blots. The levels of pT73-Rab10 proteins were normalized to $\beta$-actin levels and the results are shown as a graph. (C) LRRK2 kinase activity after serum starvation (S.S) and 100 nM MLi2 treatment for $24 \mathrm{~h}$. LRRK2 kinase activity was analyzed by the same method as Fig. Bii. (D) The genotype of each cell type was confirmed by DNA sequencing. (E) An NSC pair derived from iPSCs that were prepared from the same LRRK2 $\mathrm{G}_{2019 \mathrm{~S}^{+/}}$and WT fibroblasts were analyzed for ciliogenesis after $100 \mathrm{nM} \mathrm{MLi}-2$ treatment for $24 \mathrm{~h}$. A summary graph and a representative image for each cell type without MLi-2 treatment are shown. A total of 98 125 cells in four images were analyzed. Scale bar: $20 \mu \mathrm{m}$. The white arrow indicates a ciliated cell. ${ }^{*} \mathrm{p}<0.05,{ }^{* *} \mathrm{p}<0.01,{ }^{* * *} \mathrm{p}<0.001,{ }^{* * *} \mathrm{p}<0.0001$ by ANOVA. ${ }^{\# \#} \mathrm{p}<0.001$ by the $t$-test.

fibroblasts unexpectedly showed a significant $\sim 13$-fold increase in ciliated cells compared to the WT cells (Fig. 3A). Most previous studies were carried out under serum starvation, and reported that a decrease in LRRK2 kinase activity increased ciliogenesis in MEF and other cells $[11,14,16]$. When we cultured the cells under serum starvation without MLi-2, the G2019S fibroblasts again showed a $\sim 5$-fold (from 13 to $67 \%$ ) increase in ciliated cells compared to the WT and the difference was maintained even after MLi-2 treatment, although the fold-difference was smaller (Fig. $3 \mathrm{~B})$. In contrast, MLi-2 treatment alone produced a nonsignificant slight increase within the same genotype by ANOVA, although the difference was significant in WT by the $t$-test analysis (Fig. 3Bi). The effect of MLi-2 and the higher kinase activity of G2019S were confirmed by the comparison of pRab10 levels (Fig. 3Bii). The combined results in Figures $3 \mathrm{~A}$ and $3 \mathrm{~B}$ show that serum starvation itself increased ciliogenesis $\sim 6$-fold in each genotype as expected, but ciliogenesis in the G2019S cells was unexpectedly higher than that in the WT cells.

Serum starvation and a decrease of LRRK2 kinase activity have been reported to increase ciliogenesis in various cell types [11, 14]. Therefore, we assumed that serum starvation would decrease LRRK2 kinase activity and tested it in the MRC5 cell line. WT fibroblasts were exposed to serum starvation and MLi-2 treatment for $24 \mathrm{~h}$, and the cell lysates were subjected to Western blot analysis using the pRab10 antibody. The results showed that LRRK2 kinase activity was significantly decreased to $\sim 60 \%$ after serum starvation, and to $17 \sim 19 \%$ after treatment with MLi-2, the positive control (Fig. 3C), confirming that serum starvation indeed decreased LRRK2 kinase activity. Because our ciliogenesis result was strikingly different from the previous reports, we reconfirmed each genotype by DNA sequencing of PCR products spanning a region including the G2019S mutation (Fig. 3D).

In addition, iPSCs that originated from the same WT/G2019S fibroblast pair were differentiated into neurons via NSCs [24], 
A

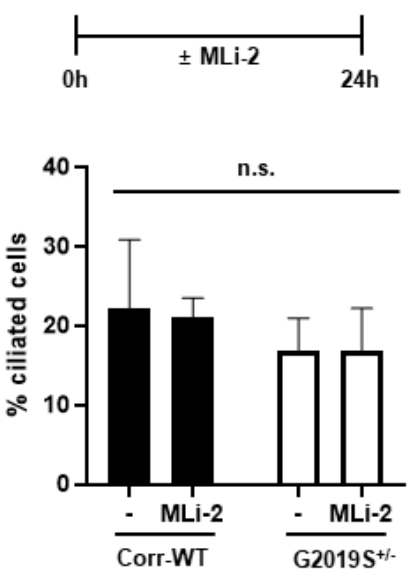

B

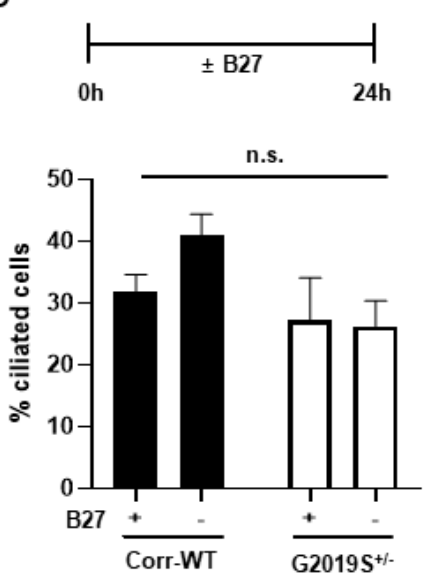

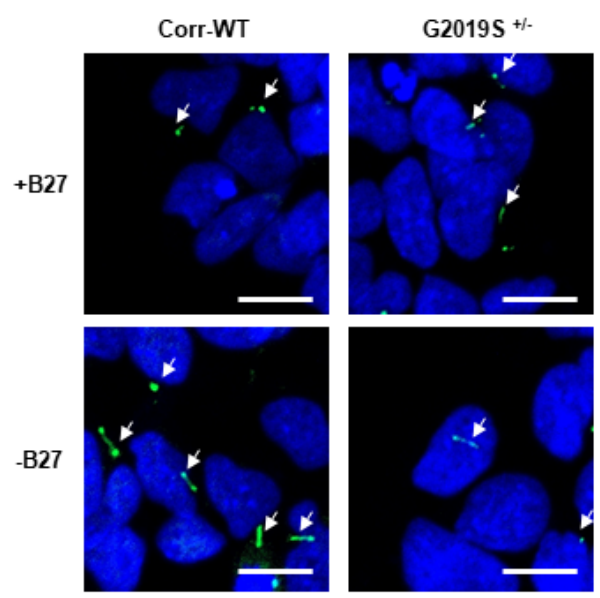

Human NSCs

Fig. 4. Ciliogenesis of isogenic LRRK2 G2019S ${ }^{+/}$and its gene-corrected WT (Corr-WT) iPSC (A) and NSC (B) pairs. iPSCs were treated with DMSO (-) or $400 \mathrm{nM}$ MLi-2 for $24 \mathrm{~h}$ (A) and NSC were exposed to B27 deprivation for $24 \mathrm{~h}$ to mimic serum starvation (B). Note that B27 deprivation could not remove all proteins because of other supplements in the medium. The treatment scheme, summary graph, and a representative image for each cell type are shown. Scale bar: $20 \mu \mathrm{m}$. A total of 106 141 (A) or 75 201 cells (B) in two (A) or four (B) images were analyzed. The white arrow indicates a ciliated cell.

and tested their ciliogenesis with or without MLi-2 treatment. The percentages of ciliated cells in the differentiated neurons were too low (less than 2\%) to conduct meaningful analysis regardless of the genotypes or MLi-2 treatment for reasons that were not clear (data not shown). We then counted the ciliated NSC cells and observed a result similar to the one in the parental fibroblasts. The ciliogenesis in G2019S cells significantly increased from 7 to $48 \%$ and from 17 to $46 \%$ compared to the WT cells without and with MLi-2, respectively, whereas MLi-2 treatment alone resulted in no significant difference in either genotype (Fig. 3E).

Dhekne et al. [14] reported that G2019S ${ }^{+/}$iPSCs originating from the fibroblasts of a patient with a heterozygous G2019S mutation exhibited a significant decrease in ciliogenesis, from $33 \%$ to $15 \%$, compared to its gene-corrected WT iPSCs, and that the MLi-

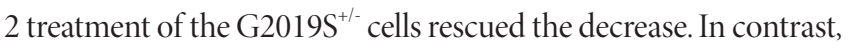
the genetic backgrounds of our WT/G2019S ${ }^{+/-}$fibroblast pair were different from each other (Fig. 3). To test whether the difference between our study and their study was due to the difference in the cellular genetic backgrounds, we utilized the previously reported

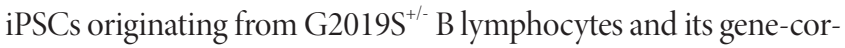
rected WT iPSCs [25]. The higher kinase activity of the G2019S iPSCs compared to the corrected WT counterparts was previously proven by increased levels of pRab10 in the G2019S cells [34]. The corrected pair of iPSCs showed 16 22\% of ciliogenesis with no significant difference regardless of the genotype or MLi2 treatment (Fig. 4A). We also tested the effect of B27 deprivation in differentiated NSCs and obtained no significant difference regardless of the genotype and B27 deprivation (Fig. 4B). Unlike the previous WT/G2019S pair (Fig. 3), the isogenic WT/G2019S +- $^{+-}$ pair exhibited no significant difference regardless of the genotype or B27 deprivation, maintaining 26 40\% ciliated cells although a slight nonsignificant increase was observed in the WT cells after B27 deprivation (Fig. 4B).

\section{Little effect of Rab10 overexpression on ciliogenesis in a dopaminergic neuronal cell line}

Rab10, an LRRK2 kinase substrate, has been reported to be a ciliogenesis suppressor because its expression decreased ciliogenesis in HEK 293 and A549 cells [14, 17]. We tested whether the pattern was also observed in neuronal cells. We transfected SN4741 murine dopaminergic neuronal model cell line with green fluorescent protein (GFP) or GFP-Rab10 WT and tested their ciliogenesis under serum starvation. We used only Rab10 WT and not phosphomimic (T73D) or -dead (T73A) mutants because these mutants have been reported to be non-functional [14]. The transfection of each cell was confirmed by its fluorescence, and the ciliated cells were immunostained using the Arl13B antibody. $\mathrm{GFP}^{+} / \mathrm{Arl}_{13 \mathrm{~B}}{ }^{+}$ cells and $\mathrm{GFP}^{+} / \mathrm{Arl} 13 \mathrm{~B}$ cells were counted separately. As a control, $\mathrm{GFP} / \mathrm{Arl}_{13 \mathrm{~B}^{+}}$cells were also counted as untransfected ciliated cells (none in Fig. 5A). The expression of both GFP and GFP-Rab10 was also confirmed by Western blots (Fig. 5B). The results showed that ciliogenesis was similar among the samples transfected or non-transfected and the overexpression of GFP-Rab10 or control GFP caused no change in ciliogenesis, which was about $38 \%$. 
A

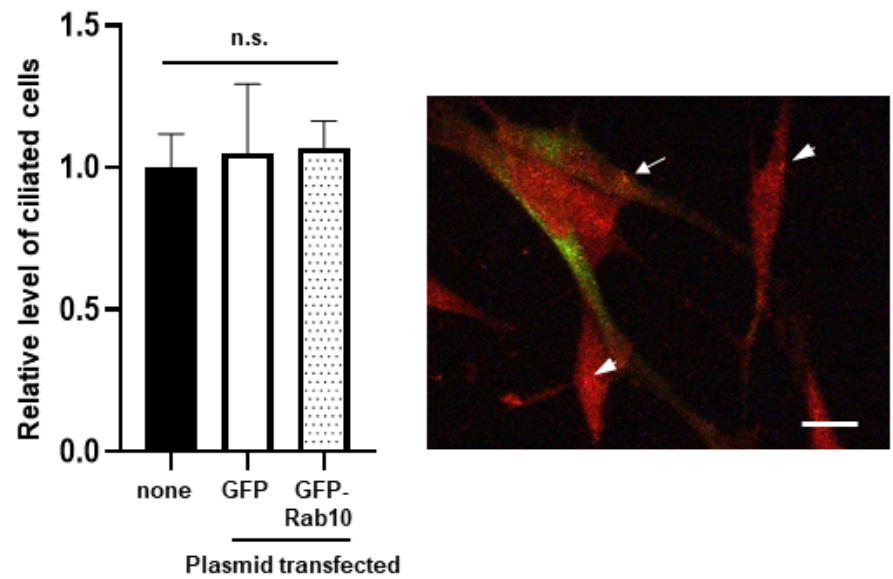

B

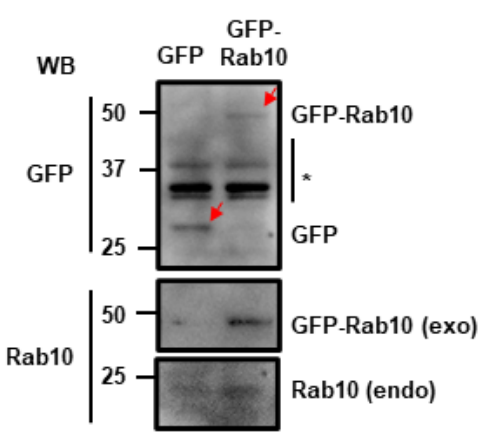

Fig. 5. Effect of Rab10 on ciliogenesis in SN4741 cells. (A) The cells were transfected with GFP or GFP-Rab10 plasmids and after a day, they were exposed to serum starvation for $24 \mathrm{~h}$. (A) Ciliated cells were stained with Arl13B (red) antibody and Arl13B ${ }^{+}$ciliated cells were counted in non-transfected (none) and $\mathrm{GFP}^{+}$or GFP Rab10 ${ }^{+}$transfected cells (green). A total of 320 413 cells in ten images were analyzed. A representative image of double-stained GFP-Rab10 transfected cells is shown. GFP-Rab10 cells transfected and non-transfected with cilia are indicated by an arrow and arrowheads, respectively. The transfection efficiency of GFP and GFP-Rab10 was 32 and 47\%, respectively, and the average was 40\%. Scale bar: $50 \mu \mathrm{m}$. B. Western blot analysis of each transfected cell type. The cell lysates were subjected to Western blot analysis with GFP and Rab10 antibodies. GFP antibody detected both GFP and GFP-Rab10 (red arrow) whereas Rab10 antibody detected both endogenous (endo) Rab10 and exogenous (exo) GFP-Rab10 proteins. *Indicates a nonspecific band. n.s.: not significant.

\section{DISCUSSION}

Our study started from a simple question on how LRRRK2 kinase activity affected on ciliogenesis in neurons. To answer it, we used various types of cells such as rat or mouse primary neurons and differentiated SH-SY5Y cells, and compared WT and G2019S genotype pairs of human fibroblasts as well as iPSCs and NSCs, and an isogenic WT/G2019S pair. In addition, various conditions such as serum starvation, B27 deprivation or MLi-2 treatment were applied.

Previous studies have repeatedly and clearly reported that LRRK2 pathogenic mutations such as G2019S or R1441C/ $G$ reduced ciliogenesis and LRRK2 kinase inhibitor treatment increased ciliogenesis, suggesting that LRRK2 kinase activity negatively regulates ciliogenesis $[11,14,15,17]$. However, our results suggest that the relationship between LRRK2 kinase activity and ciliogenesis is not as simple as previously reported. We could detect little difference in ciliogenesis between WT and G2019S genotypes (Fig. 2, 4). The only significant difference was observed in WT and G2019S ${ }^{+/-}$fibroblasts and NSCs (Fig. 3), which showed an increase in ciliogenesis in $\mathrm{G}_{2019 S^{+/-}}$cells, opposite the results in previous reports. We reproducibly observed more than a $\sim$-fold increase in ciliogenesis in $\mathrm{G}^{2} 019 \mathrm{~S}^{+/-}$fibroblasts compared to WT (Fig. 3A) and similar results were obtained in a separate laboratory when the same cell pair was used. Decreases and increases in LRRK2 kinase activity by MLi-2 treatment and in the G2019S genotype, respectively, were confirmed by p T73-Rab10 levels in Western blot analysis of the corresponding cell lysates (Fig. 3Bii). In contrast to the fibroblast and NSC results in Figure 3, little difference in ciliogenesis was observed between primary neurons prepared from G2019S ${ }^{+/-}$and WT littermate mice (Fig. 2) and in another human WT/G2019S pair under an isogenic background (Fig. 4). Our data suggested that the cell type, as well as the genetic or species background, is more critical to determining ciliogenesis than the LRRK2 kinase activity.

Another difference was the small effect of LRRK2 kinase inhibitor treatment on ciliogenesis, suggesting again weak correlation between LRRK2 kinase activity and ciliogenesis. In most cases, MLi-2 treatment resulted in little difference in both WT and the pathogenic G2019S genotype (Figs. 1, 3, 4), although a weak increase was observed in ciliogenesis in dSH-SY5Y cells (Fig. 1A) and WT fibroblasts (Fig. 3B). It is worth noting that Lara Ordónez et al. [17] observed an increasing effect on ciliogenesis by MLi-2 treatment only in HEK 293 T cells expressing LRRK2 pathogenic mutations, but not WT, under serum-fed conditions.

A few points can be considered the reasons for the difference in WT vs. G2019S results between our study and previous studies [14, 17]. The most probable explanation is that the regulation of ciliogenesis might be primarily genetic- and/or cell type-specific [35]. Ciliogenesis induced by serum starvation was observed in several cell types [9]. However, one study reported that HEK293T cells exhibited no stimulation of ciliogenesis by serum starvation and 
there was no difference between WT and G2019S knock-in mice astrocytes under serum starvation condition regardless of MLi2 treatment [17]. In addition, a deficit in ciliogenesis in R1441C mice was reported only in $\mathrm{ChAT}^{+}$but not in ChAT neurons of the striatum [14]. We also observed an increase of ciliogenesis in one

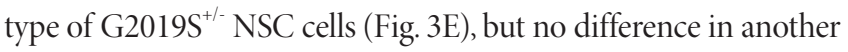
type of G2019S ${ }^{+-}$NSC cells, compared to the WT NSCs (Fig. 4B). This was probably due to the difference in LRRK2 expression levels among the tissues we used. The LRRK2 expression level is the highest in B-lympocytes that was used to prepare the isogenic WT/G2019S pair used in Fig. 4, and was similar between the cortex, hippocampus and skin when the levels were compared based on the Protein Atlas (https://www.genecards.org/cgi-bin/carddisp. pl?gene=LRRK2) and Gene-Cards (https://www.genecards.org/
cgi-bin/carddisp.pl?gene=LRRK2). Taken together, these results suggest that genetic backgrounds and the cellular expression patterns of proteins affecting basal LRRK2 kinase activity such as phosphatase PPM1H [15] are the critical determinants of ciliogenesis rather than the LRRK2 expression level. A recent study reported that the genetic backgrounds of rats resulted in different pT73-Rab10 levels despite similar LRRK2 expression levels [36]. Rab8 and Rab10 might be such proteins affecting ciliogenesis because they function in the opposite ways in ciliogenesis although both Rab proteins are LRRK2 kinase substrates [14]. In addition, the genetic background may differ by single base due to single nucleotide polymorphisms present in the LRRK2 gene itself used in each study. Although many amino acid variations in LRRK2 had been reported $[37,38]$ and their physiological roles are not

Table 1. Summary of studies on the relationship between LRRK2 and ciliogenesis

\begin{tabular}{|c|c|c|c|c|c|}
\hline Study & Tissue or cell line & Targets investigated & Cilia marker & $\begin{array}{c}\text { Ciliogenesis change by the } \\
\text { treatments }\end{array}$ & Reference \\
\hline \multirow{21}{*}{ Previous } & A549, RPE & Rab10KO & & $\uparrow$ by Rab10KO* & \multirow{7}{*}{14} \\
\hline & MEF-LRRK2-R1441G & MLi-2 treatment and Rab10KD & & $\uparrow$ by $\mathrm{MLi}^{-} 2^{\star}$ and by Rab10KD ${ }^{*}$ & \\
\hline & IPS cells & LRRK $2^{\text {WT/WT }}$ vs LRRK2 $2^{\mathrm{G} 2019 \mathrm{~S} / \mathrm{WT}}$ & Arl13B & $\downarrow$ in LRRK2 $2^{\mathrm{G} 2019 \mathrm{~S} / \mathrm{NT}}$ & \\
\hline & LRRK2 $^{\mathrm{G} 2019 \mathrm{~S} / \mathrm{WT}}$ IPS cells & MLi-2 treatment & & $\uparrow$ by $\mathrm{MLi}-2^{\star}$ & \\
\hline & Neurons in the cortex & \multirow{3}{*}{ WT/WT vs R1441C/R1441C } & SSTR & $\downarrow$ in LRRK2 $2^{\mathrm{R} 1441 \mathrm{C} / \mathrm{R} 1441 \mathrm{C}_{\star}}$ & \\
\hline & Cholinergic neurons in the striatum & & \multirow{2}{*}{$\mathrm{AC} 3$} & $\downarrow$ in LRRK2 $2^{\text {R144lC/R1441C }}$ & \\
\hline & Non-cholinergic neurons in the striatum & & & - by MLi-2 & \\
\hline & $\begin{array}{l}\text { HEK } 293 \text { cells transfected with vector or LRRK2 } \\
\text { WT }\end{array}$ & \multirow{2}{*}{ MLi-2 treatment } & \multirow{3}{*}{ PT } & - by MLi-2 & \multirow{6}{*}{17} \\
\hline & $\begin{array}{l}\text { HEK } 293 \text { cells expressing LRRK2 G2019S, } \\
\text { R1441C, Y1699C }\end{array}$ & & & $\uparrow$ by MLi- $2^{\#}$ & \\
\hline & Human fibroblast & LRRK2 WT vs G2019S & & $\downarrow$ in LRRK2 $2^{\mathrm{G} 2019 \mathrm{~S} / \mathrm{WT} T_{\star}}$ & \\
\hline & WT \& G2019S human fibroblast & \multirow{3}{*}{ MLi-2 treatment } & PT & - by MLi-2 & \\
\hline & WT primary astrocytes & & \multirow{2}{*}{ Arl13B } & - by MLi-2 & \\
\hline & G2019S KI primary astrocytes & & & 个by MLi-2" & \\
\hline & SH-SY5Y & Differentiation & \multirow{3}{*}{$\mathrm{AT}$} & $\uparrow$ by differentiation & \multirow{3}{*}{39} \\
\hline & $\begin{array}{l}\text { SH-SY5Y (both differentiated and undifferenti- } \\
\text { ated) }\end{array}$ & MG132 treatment & & $\downarrow$ by MG132* & \\
\hline & Undifferentiated SH-SY5Y & $\begin{array}{l}\text { a-synuclein WT, A30P, A53T } \\
\text { expression }\end{array}$ & & $\downarrow$ by $a$-synuclein expression ${ }^{*}$ & \\
\hline & SH-SY5Y & Rotenone, $\mathrm{MPP}^{+}$treatment & Arl13B & $\uparrow$ by treatments ${ }^{\#}$ & 20 \\
\hline & MEF & Scrambled shRNA & Arl13B & - by Mli-2** & 15 \\
\hline & MEF-LRRK2-R1441C & MLi-2 treatment & SSTR3-GFP & $\uparrow$ by MLi-2* & 16 \\
\hline & MEF-LRRK2-R1441G KI & ML: 2 troctmont & $\mathrm{HaP}$ & 个 & 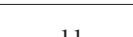 \\
\hline & NIH3T3 transfected with LRRK2 G2019S & ML1-2 treatment & Arl13B & T by ML1-2 & 11 \\
\hline \multirow{9}{*}{ Our study } & Rat cortical/ hippocampal WT primary neurons & GSK/MLi-2 treatment & \multirow{9}{*}{ Arl13B } & - by treatment & \multirow{9}{*}{ This study } \\
\hline & Fibroblasts (LRRK2 $2^{\mathrm{G} 2019 \mathrm{~S} / \mathrm{WT}}$ ) & MLi-2 treatment & & - by MLi-2 & \\
\hline & Fibroblasts (LRRK2 ${ }^{\mathrm{WT} / \mathrm{WT}}{ }_{\text {VS }}$ LRRK2 ${ }^{\mathrm{G} 2019 \mathrm{~S} / \mathrm{WT} \mathrm{T}}$ ) & & & $\uparrow$ in LRRK2 $2^{\mathrm{G} 20195 / W T \# \#}$ & \\
\hline & NSCs (LRRK2 ${ }^{\text {WT/WT }}$ vs LRRK2 $\left.2^{\text {G2019S/WT }}\right)$ & LRRK $2^{\text {WT/WT }}$ Vs LRRK2 $2^{\text {G2019S/WT }}$ & & $\uparrow$ in LRRK2 $2^{\mathrm{G} 2019 \mathrm{~S} / \mathrm{WT} \# \#}$ & \\
\hline & Murine cortical primary neurons & & & - between genotypes & \\
\hline & \multirow{2}{*}{$\begin{array}{l}\text { Isogenic pairs of iPSCs and NSCs (LRRK2 }{ }^{\text {WT/WT }} \\
\text { vs LRRK2 } 2^{\text {G2019S/WT }} \text { ) }\end{array}$} & $\mathrm{LRRK}^{\mathrm{WTTWT}}{ }_{\text {VS }} \mathrm{LRRK} 2^{\mathrm{G} 2019 \mathrm{~S} / \mathrm{WT}}$ & & - between genotypes & \\
\hline & & MLi-2 treatment & & - by MLi-2 & \\
\hline & \multirow{2}{*}{ Differentiated SH-SY5Y } & MLi-2 treatment & & Slightly $\uparrow$ by MLi- $2^{\# \#}$ & \\
\hline & & Rab10WT expression & & - by expression & \\
\hline
\end{tabular}

-, little change; KI, Knock-in; AC3, adenylate cyclase 3; AT, acetylated tubulin; PT, polyglutamylated tubulin; SSTR, somatostatin receptor.

${ }^{*}$ Statistically significant change.

** The result was observed in the control sample (scrambled shRNA) to test effect of PPM1H knockdown on ciliogenesis.

"Statistically significant change was observed only under normal serum-fed condition, but not in the serum starved condition.

*A Significant change regardless of serum condition. 
clear yet, most studies only confirmed the pathogenic mutations themselves by DNA sequencing.

We found that a considerable number of cells contained cilia in dSH-SY5Y, murine primary neurons and G2019S fibroblasts under serum-starved conditions (Figs. 1A, 2, 3B). The lack of effect by serum starvation, B27 deprivation or MLi-2 treatment might be due to the high rate of ciliogenesis in neurons. However, it is worth noting that -20 -30\% ciliogenesis in rat primary neurons and $\mathrm{iP}$ SCs of the G2019S/corrected WT isogenic pair was not increased after MLi-2 treatment (Figs. 1B, 4A).

To easily compare the previous results with our results, a summary is presented in Table 1.

Taken together, we observed no negative effect of LRRK2 kinase activity on ciliogenesis in neurons but an increase in ciliogenesis in the G2019S fibroblast cell line, opposite to many previous reports $[11,14-18,39]$. Our data recommend a careful approach to investigating the relationship between LRRK2 kinase activity and ciliogenesis and suggest that the genetic background must be considered to explain the relationship between LRRK2 kinase activity and ciliogenesis. To clarify the discrepant effect of LRRK2 kinase activity on ciliogenesis, it may be necessary to investigate ciliogenesis in several independent LRRK2 WT/mutant cells or G2019S/ WT isogenic pairs.

\section{ACKNOWLEDGEMENTS}

We thank Dr. Ho, DH for his suggestion and help. This study was supported by the Basic Science Research Program through the National Research Foundation (NRF) funded by the Ministry of Education, Science and Technology, Republic of Korea (WS:2018R1D1A1B07041153; SKC:NRF-2013M3A9B4076487), the grant (JK:18721MFDS182) from the Ministry of Food and Drug Safety, and the KRIBB research initiative program (JK:KGM5362111) funded by the Ministry of Science and ICT.

\section{CONFLICT OF INTEREST}

The authors declare no conflict of interest.

\section{REFERENCES}

1. Seol W (2010) Biochemical and molecular features of LRRK2 and its pathophysiological roles in Parkinson's disease. BMB Rep 43:233-244.

2. Cookson MR (2017) Mechanisms of mutant LRRK2 neurodegeneration. Adv Neurobiol 14:227-239.

3. Monfrini E, Di Fonzo A (2017) Leucine-rich repeat kinase
(LRRK2) genetics and Parkinson's disease. Adv Neurobiol 14:3-30.

4. West AB, Moore DJ, Biskup S, Bugayenko A, Smith WW, Ross CA, Dawson VL, Dawson TM (2005) Parkinson's diseaseassociated mutations in leucine-rich repeat kinase 2 augment kinase activity. Proc Natl Acad Sci U S A 102:16842-16847.

5. Lesage S, Dürr A, Tazir M, Lohmann E, Leutenegger AL, Janin S, Pollak P, Brice A; French Parkinson's Disease Genetics Study Group (2006) LRRK2 G2019S as a cause of Parkinson's disease in North African Arabs. N Engl J Med 354:422-423.

6. Zhao Y, Dzamko N (2019) Recent developments in LRRK2targeted therapy for Parkinson's disease. Drugs 79:1037-1051.

7. Lee JH, Gleeson JG (2010) The role of primary cilia in neuronal function. Neurobiol Dis 38:167-172.

8. Gazea M, Tasouri E, Tolve M, Bosch V, Kabanova A, Gojak C, Kurtulmus B, Novikov O, Spatz J, Pereira G, Hübner W, Brodski C, Tucker KL, Blaess S (2016) Primary cilia are critical for Sonic hedgehog-mediated dopaminergic neurogenesis in the embryonic midbrain. Dev Biol 409:55-71.

9. Santos N, Reiter JF (2008) Building it up and taking it down: the regulation of vertebrate ciliogenesis. Dev Dyn 237:19721981.

10. Steger M, Tonelli F, Ito G, Davies P, Trost M, Vetter M, Wachter S, Lorentzen E, Duddy G, Wilson S, Baptista MA, Fiske BK, Fell MJ, Morrow JA, Reith AD, Alessi DR, Mann M (2016) Phosphoproteomics reveals that Parkinson's disease kinase LRRK2 regulates a subset of Rab GTPases. Elife 5:e12813.

11. Steger M, Diez F, Dhekne HS, Lis P, Nirujogi RS, Karayel O, Tonelli F, Martinez TN, Lorentzen E, Pfeffer SR, Alessi DR, Mann M (2017) Systematic proteomic analysis of LRRK2mediated Rab GTPase phosphorylation establishes a connection to ciliogenesis. Elife 6:e31012.

12. Yoshimura S, Egerer J, Fuchs E, Haas AK, Barr FA (2007) Functional dissection of Rab GTPases involved in primary cilium formation. J Cell Biol 178:363-369.

13. Sato T, Iwano T, Kunii M, Matsuda S, Mizuguchi R, Jung Y, Hagiwara H, Yoshihara Y, Yuzaki M, Harada R, Harada A (2014) Rab8a and Rab8b are essential for several apical transport pathways but insufficient for ciliogenesis. J Cell Sci 127(Pt 2):422-431.

14. Dhekne HS, Yanatori I, Gomez RC, Tonelli F, Diez F, Schüle B, Steger M, Alessi DR, Pfeffer SR (2018) A pathway for Parkinson's disease LRRK2 kinase to block primary cilia and Sonic hedgehog signaling in the brain. Elife 7:e40202.

15. Berndsen K, Lis P, Yeshaw WM, Wawro PS, Nirujogi RS, Wightman M, Macartney T, Dorward M, Knebel A, Tonelli F, Pfeffer SR, Alessi DR (2019) PPM1H phosphatase counter- 
acts LRRK2 signaling by selectively dephosphorylating Rab proteins. Elife 8:e50416.

16. Sobu Y, Wawro PS, Dhekne HS, Yeshaw WM, Pfeffer SR (2021) Pathogenic LRRK2 regulates ciliation probability upstream of tau tubulin kinase 2 via Rab10 and RILPL1 proteins. Proc Natl Acad Sci U S A 118:e2005894118.

17. Lara Ordónez AJ, Fernández B, Fdez E, Romo-Lozano M, Madero-Pérez J, Lobbestael E, Baekelandt V, Aiastui A, López de Munaín A, Melrose HL, Civiero L, Hilfiker S (2019) RAB8, RAB10 and RILPL1 contribute to both LRRK2 kinase-mediated centrosomal cohesion and ciliogenesis deficits. Hum Mol Genet 28:3552-3568.

18. Dhekne HS, Yanatori I, Vides EG, Sobu Y, Diez F, Tonelli F, Pfeffer SR (2021) LRRK2-phosphorylated Rab10 sequesters Myosin Va with RILPL2 during ciliogenesis blockade. Life Sci Alliance 4:e202101050.

19. Xicoy H, Wieringa B, Martens GJ (2017) The SH-SY5Y cell line in Parkinson's disease research: a systematic review. Mol Neurodegener 12:10.

20. Bae JE, Kang GM, Min SH, Jo DS, Jung YK, Kim K, Kim MS, Cho DH (2019) Primary cilia mediate mitochondrial stress responses to promote dopamine neuron survival in a Parkinson's disease model. Cell Death Dis 10:952.

21. Son MY, Sim H, Son YS, Jung KB, Lee MO, Oh JH, Chung SK, Jung CR, Kim J (2017) Distinctive genomic signature of neural and intestinal organoids from familial Parkinson's disease patient-derived induced pluripotent stem cells. Neuropathol Appl Neurobiol 43:584-603.

22. Liu GH, Qu J, Suzuki K, Nivet E, Li M, Montserrat N, Yi F, Xu X, Ruiz S, Zhang W, Wagner U, Kim A, Ren B, Li Y, Goebl A, Kim J, Soligalla RD, Dubova I, Thompson J, Yates J 3rd, Esteban CR, Sancho-Martinez I, Izpisua Belmonte JC (2012) Progressive degeneration of human neural stem cells caused by pathogenic LRRK2. Nature 491:603-607.

23. Sim H, Seo JH, Kim J, Oh M, Lee JE, Baek A, Lee SY, Chung SK, Son MY, Chae JI, Jeon YJ, Kim J (2020) Quantitative proteomic analysis of primitive neural stem cells from LRRK2 G2019S-associated Parkinson's disease patient-derived iPSCs. Life (Basel) 10:331.

24. Sim H, Lee JE, Yoo HM, Cho S, Lee H, Baek A, Kim J, Seo H, Kweon MN, Kim HG, Jeon YJ, Son MY, Kim J (2020) Iroquois homeobox protein 2 identified as a potential biomarker for Parkinson's disease. Int J Mol Sci 21:3455.

25. Lee SY, Chung SK (2019) Generation of gene-corrected iPSC line, KIOMi002-A, from Parkinson's disease patient iPSC with LRRK2 G2019S mutation using BAC-based homolo- gous recombination. Stem Cell Res 41:101649.

26. Park SW, Mansur RB, Lee Y, Lee JH, Seo MK, Choi AJ, McIntyre RS, Lee JG (2018) Liraglutide activates mTORC1 signaling and AMPA receptors in rat hippocampal neurons under toxic conditions. Front Neurosci 12:756.

27. Ho DH, Je AR, Lee H, Son I, Kweon HS, Kim HG, Seol W (2018) LRRK2 kinase activity induces mitochondrial fission in microglia via Drpl and modulates neuroinflammation. Exp Neurobiol:171-180.

28. Berbari NF, Bishop GA, Askwith CC, Lewis JS, Mykytyn K (2007) Hippocampal neurons possess primary cilia in culture. J Neurosci Res 85:1095-1100.

29. King CR, A A Quadros AR, Chazeau A, Saarloos I, van der Graaf AJ, Verhage M, Toonen RF (2019) Fbxo41 promotes disassembly of neuronal primary cilia. Sci Rep 9:8179.

30. Pierce NW, Nachury MV (2013) Cilia grow by taking a bite out of the cell. Dev Cell 27:126-127.

31. Pang T, Sun LX, Wang T, Jiang ZZ, Liao H, Zhang LY (2014) Telmisartan protects central neurons against nutrient deprivation-induced apoptosis in vitro through activation of PPAR $\gamma$ and the Akt/GSK-3 $\beta$ pathway. Acta Pharmacol Sin 35:727-737.

32. Brewer GJ, Torricelli JR, Evege EK, Price PJ (1993) Optimized survival of hippocampal neurons in B27-supplemented Neurobasal, a new serum-free medium combination. J Neurosci Res 35:567-576.

33. Young JE, Martinez RA, La Spada AR (2009) Nutrient deprivation induces neuronal autophagy and implicates reduced insulin signaling in neuroprotective autophagy activation. J Biol Chem 284:2363-2373.

34. Ha J, Kang JS, Lee M, Baek A, Kim S, Chung SK, Lee MO, Kim J (2020) Simplified brain organoids for rapid and robust modeling of brain disease. Front Cell Dev Biol 8:594090.

35. Wiegering A, Dildrop R, Kalfhues L, Spychala A, Kuschel S, Lier JM, Zobel T, Dahmen S, Leu T, Struchtrup A, Legendre F, Vesque C, Schneider-Maunoury S, Saunier S, Rüther U, Gerhardt C (2018) Cell type-specific regulation of ciliary transition zone assembly in vertebrates. EMBO J 37:e97791.

36. Kelly K, Chang A, Hastings L, Abdelmotilib H, West AB (2021) Genetic background influences LRRK2-mediated Rab phosphorylation in the rat brain. Brain Res 1759:147372.

37. Shin N, Jeong H, Kwon J, Heo HY, Kwon JJ, Yun HJ, Kim CH, Han BS, Tong Y, Shen J, Hatano T, Hattori N, Kim KS, Chang S, Seol W (2008) LRRK2 regulates synaptic vesicle endocytosis. Exp Cell Res 314:2055-2065.

38. Mata IF, Kachergus JM, Taylor JP, Lincoln S, Aasly J, Lynch T, 
Hulihan MM, Cobb SA, Wu RM, Lu CS, Lahoz C, Wszolek ZK, Farrer MJ (2005) Lrrk2 pathogenic substitutions in Parkinson's disease. Neurogenetics 6:171-177.
39. Iqbal A, Baldrighi M, Murdoch JN, Fleming A, Wilkinson CJ (2020) Alpha-synuclein aggresomes inhibit ciliogenesis and multiple functions of the centrosome. Biol Open 9:bio054338. 